\title{
Compression and deformation of soft spherical particles
}

\author{
Yu-Li Lin ${ }^{\mathrm{a}}$, Da-Ming Wang ${ }^{\mathrm{a}}$, Wei-Ming Lu ${ }^{\mathrm{a}}$, Yu-Shen Lin ${ }^{\mathrm{b}}$, Kuo-Lun Tung ${ }^{\mathrm{b}, *}$ \\ ${ }^{a}$ Department of Chemical Engineering, National Taiwan University, Taipei 106, Taiwan \\ ${ }^{\mathrm{b}}$ Department of Chemical Engineering, R\&D Center for Membrane Technology, Chung Yuan Christian University, Chungli 320, Taiwan
}

Received 18 March 2007; received in revised form 21 July 2007; accepted 14 September 2007

Available online 21 September 2007

\begin{abstract}
In order to model geometric property variation and investigate different compression behaviors between compressible (variable volume) and incompressible (invariable volume) soft spherical particles, two compression models based on different shapes of the lateral surface (noncontacted surface of the particle) of the compressed particles are proposed. The shape profiles in various compression degrees calculated by the models showed good agreement with the experimental data. The models can be also used to estimate the surface area and volume of the soft particles. Additionally, according to particle shape profiles, the particle structures, porous or dense, show great influences on the compression behavior for both compressible and incompressible soft particles, where the dense incompressible particle performs a higher degree of lateral extension during compression. This is because its volume can be transferred more completely from the compressed portion to the lateral surface, which is without loading contact on it.
\end{abstract}

(C) 2007 Elsevier Ltd. All rights reserved.

Keywords: Deformation; Compressible; Incompressible; Soft particle; Uni-axial compression

\section{Introduction}

Soft particles, including microorganisms, emulsions, gels, etc., are usually encountered in food, bio-technology, and biomedical industries, and are also major chromatography process components. The deformability of the soft particle is the key characteristic, involving manufacturing, separation, and purification of products containing such material. Soft particle deformation is a behavior that concerns change of shape, mechanical properties, or even volume variation in the particle. By this concept, soft particles can be roughly categorized into two types: (1) The overall volume of an "incompressible" soft particle can stay constant during a compression process. It means that even if the shape of the particle is varied, the magnitude of its volume remains unchanged. Rubber can approximately perform with such characteristics since rubber products can be manufactured quite densely. (2) The volume of a "compressible" soft particle is variable as being compressed. Sponge is a common compressible material that contains porous inner structures. Since there are apparent differences in the intrinsic

\footnotetext{
* Corresponding author. Tel.: +8863 2654129; fax: +88632654199

E-mail address: kuolun@cycu.edu.tw (K.-L. Tung).
}

characteristics between these particles, it is worth to understand and compare the performances between these particles under compression.

Hertz (Johnson, 1985) conducted the first specific research on soft particle compression in 1882. He studied the fully elastic sphere compressed between two flat, rigid surfaces with a small compression ratio (penetration depth/diameter of the sphere), where penetration depth is much smaller than sphere diameter. Later researchers have modified and extended the Hertz theory to a greater compression ratio range. Tatara (1993) studied rubber sphere large deformation. Based on the Hertz theory, he derived equations containing mechanical parameters (Young's modulus and Poisson's ratio) for geometric variation estimation of the rubber sphere under uni-axial compression (Tatara's theory). The Hertz theory was examined and its valid compression ratio shown to be less than $10 \%$, but in good agreement up to $80 \%$ compression ratio for the new theory proposed by Tatara (1993). Advanced modification was applied to Tatara's theory, improving its validity in the greater compression ratio range. Lacasse et al. (1996a, b) and Devlaminck (1999) proposed numerical analyses to solve the surface profile of a fixed-volume spherical droplet compressed between two parallel plates, and a method of three-dimensional simulation 
is established to model the elastic properties of a disordered emulsion. Liu et al. $(1996,1998)$ examined Hertz and Tatara's theories using the polyurethane sphere under uni-axial compression between flat rigid surfaces, and confirmed their validity under various compression ratios. Lu et al. (2001) referred to Tatara's studies and derived an equation without mechanical parameters relating compressed sphere penetration depth and lateral extension. The derived theory has been applied to analyze the compression behavior of soft particles in a packed bed under steady state condition (Tung et al., 2004).

With more complicated behaviors, ex: the variation is the internal structures related to the degree of compression, the issues of the compression behavior of the compressible soft particles are less discussed. Andrei et al. (1996) used the microscopic device to investigate compression of the polydextran Sephadex bead, which is the swollen material and particle volume that can be reduced due to water migration from the particle under compression. Findings showed that the Kelvin model could be properly used to describe the Sephadex bead compression, which exhibited a time-dependent characteristic. Regarding the timedependent problem, the model of Adams and McKeown (1996) has been widely used in the recent literature. This model has been developed based on a first order rate process relating the loading pressure to the porosity of particle bed. Furthermore, deformable particles can exhibit either elastic or elastic-plastic properties; hence both material laws are used in continuum mechanics (Adams et al., 2004; Sheng et al., 2004; Antonyuk et al., 2005). However, particle beds are individual compounds, and thus the discrete element method is better to use for calculating the contact forces and relative movements of particles (Reynolds et al., 2005). During this simulation particle bed is regarded as a system of primary particles. The discrete element computer simulations can illustrate more details of the deformation behavior (Samimi et al., 2005).

The compressible soft particles are often encountered in the daily life and various biological or medicinal products; hence applying further investigations to obtain more understanding of the compression behavior of such material is necessary. Moreover, the study of the comparison between uni-axial compressed single or multiple compressible and incompressible soft particles is conducive to find out the major differences of these material among various mechanical operations. Establishing models to estimate the change of the properties, such as the inner porosity and the contour, of the soft particle by investigating the geometric variations under uni-axial mechanical compression is the purpose of this study. Particle image observation and analysis during compression and static state are adopted to describe lateral surface profile (i.e., the surface which does not contact with the compression unit). Model equations are also used to estimate surface area variation and compressed particle volume.

\section{The model of the lateral profile}

For a soft particle under uni-axial compression, the volume of the compression path, i.e., the penetration path, will be "reduced". The reduced volume will be migrated to the

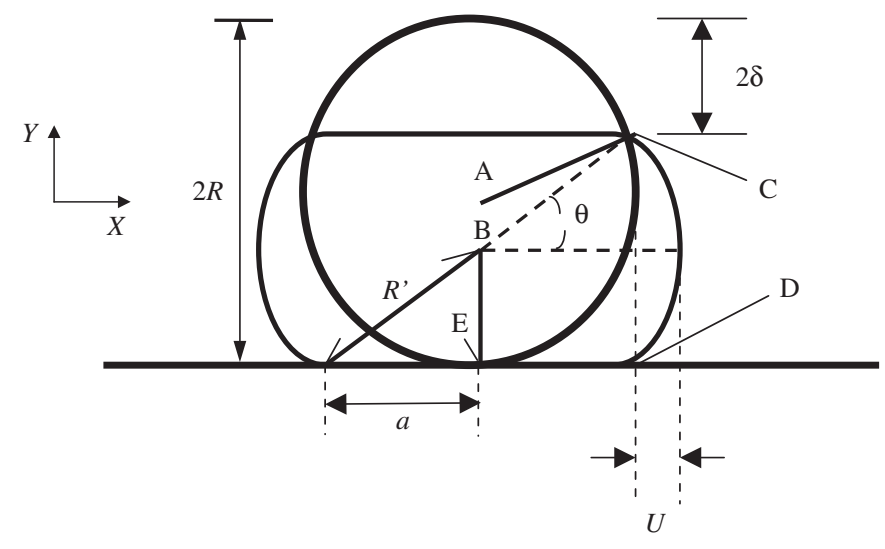

Fig. 1. The definition of the physical quantities for the spherical model.

lateral surface side, which is free of compression loading. The volume migration will then lead to the extension of the lateral surface. To obtain particle variation influence, geometric parameter models, such as the particle radius, contact area, etc., can be constructed. The assumptions of this study are listed as follows:

1. particle shape is fully spherical before loading;

2. particle inner structures, whether compressible or incompressible, are uniform;

3. the shape of the compressed particle remains axisymmetric;

4. displacement of any axisymmetric position is identical.

Fig. 1 shows the schematic diagram of a compressed spherical particle under mechanical load. Points $A$ and $B$ are the particle centers without and with compression; $C$ and $D$ are the upper and lower intersections of the contact surface and the lateral surface. $E$ is the center of the contact surface. $\delta, U$, and $a$ are the penetration depth (for semi-sphere, overall penetration depth is $2 \delta$ ), lateral extension, and radius of the contact surface respectively. $R^{\prime}$ is the center length to the lateral surface at the compressed particle.

The lateral surface profile will be assumed as spherical and elliptical shaped to construct the compression models in this study.

\subsection{Spherical lateral surface}

From Fig. 1, if the lateral surface of the compressed particle is spherical, the center length to the lateral surface is $R^{\prime}$, and the contact surface radius, $a$, and the contact area, $S$, can be calculated by the following equations:

$a=\sqrt{R^{\prime 2}-(R-\delta)^{2}}$,

$S=\pi\left[R^{\prime 2}-(R-\delta)^{2}\right]$.

Fig. 2 shows the coordinate of a differential volume on the compressed spherical particle surface. The shaded area indicates the lateral surface. As $\theta$ and $\psi \rightarrow 0$, the differential lateral 


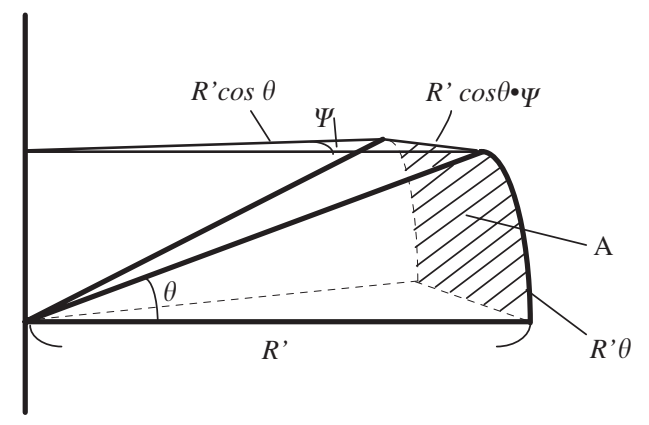

Fig. 2. The coordinate of the control volume at surface of the sphere.

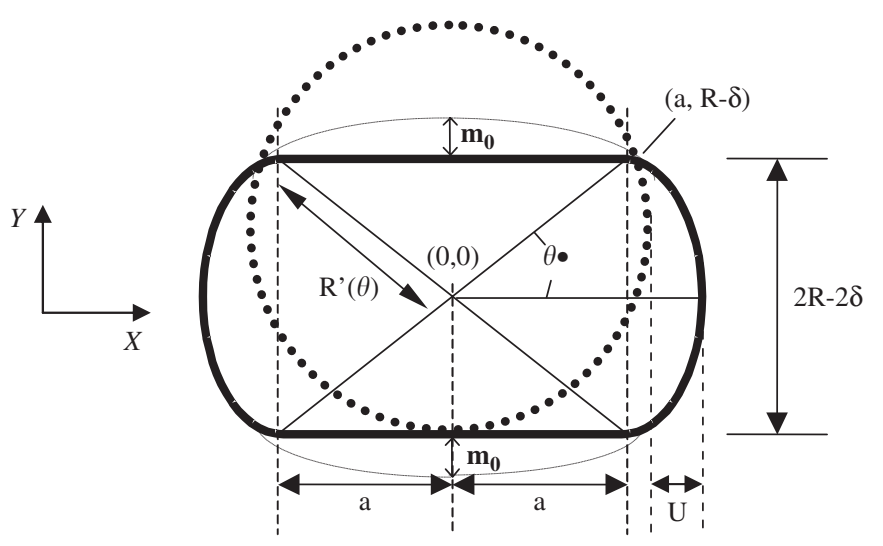

Fig. 3. The definition of the physical quantities for the elliptical model.

surface area can be calculated by the following equation:

$\mathrm{d} A=\left(R^{\prime} \mathrm{d} \theta\right) \cdot\left(R^{\prime} \cos \theta \cdot \mathrm{d} \psi\right)$.

The lateral surface area of the half-particle can be obtained by integrating Eq. (3), and the overall lateral surface area, $A_{S}$, is twice this value to be as the following:

$A_{S}=4 \pi R^{\prime 2} \sin (\theta)$,

where $\theta=\tan ^{-1} \frac{R-\delta}{\sqrt{R^{\prime 2}-(R-\delta)^{2}}}$. The volume of the compressed particle can be calculated by the following equation:

$V=2 \pi\left[R^{\prime 2}(R-\delta)-\frac{(R-\delta)^{3}}{3}\right]$.

\subsection{Elliptical lateral surface}

If the lateral surface profile is assumed as elliptical shaped, this system coordinate can be constructed as shown in Fig. 3. The visualized dash-lines in Fig. 3 link the contact and lateral surface intersections to make the compressed particle an elliptical shape, and the length between the roof of the dash line and the contact surface is $m_{0}$. The length of the semi-major and the semi-minor axes are $R+U$ and $R-\delta+m_{0}$, respectively. Insert these values into the elliptical equation, and an equation to estimate the profile of the lateral surface can be obtained:

$\frac{x^{2}}{(U+R)^{2}}+\frac{y^{2}}{\left(R-\delta+m_{0}\right)^{2}}=1$.

Take the coordinate of the intersection of the contact and lateral surface $[a,(R-\delta)]$ into Eq. (6):

$\frac{a^{2}}{(U+R)^{2}}+\frac{(R-\delta)^{2}}{\left(R-\delta+m_{0}\right)^{2}}=1$.

Rearrange the above equation to obtain the value $m_{0}$, and the elliptical model equation can be established by taking the value $m_{0}$ into Eq. (7):

$x^{2}+\left[\frac{(U+R)^{2}-a^{2}}{(R-\delta)^{2}}\right] y^{2}=(U+R)^{2}$.

To calculate the volume and surface area of the compressed particle, it is able to introduce the parameter function into the elliptical model function.

$x=A^{\prime} \cos \chi_{0}$,

$y=B^{\prime} \sin \chi_{0}$,

where $\chi_{0}$ is the variable parameter in the unit of radian. Comparing Eqs. (8) and (9) to the elliptical model function, the parameters $A^{\prime}$ and $B^{\prime}$ can be obtained:

$A^{\prime 2}=(U+R)^{2}$,

$B^{\prime 2}=\left[\frac{(U+R)^{2}(R-\delta)^{2}}{(U+R)^{2}-a^{2}}\right]$.

The value of $R^{\prime}$ can be calculated as

$R^{\prime}=\left(A^{\prime 2} \cos ^{2} \chi_{0}+B^{\prime 2} \sin ^{2} \chi_{0}\right)^{1 / 2}$.

Thus, the overall area of the lateral surface can be obtained:

$$
\begin{aligned}
A_{E}= & \frac{4 \pi A^{\prime 2} B^{\prime}}{\sqrt{A^{\prime 2}-B^{\prime 2}}} \\
& \times \sin ^{-1}\left(\sqrt{\frac{A^{\prime 2}-B^{\prime 2}}{A^{\prime 2}}} \sin \left(\tan ^{-1} \frac{A^{\prime}}{B^{\prime}} \frac{R-\delta}{a}\right)\right)
\end{aligned}
$$

and the volume of the particle will be

$$
\begin{aligned}
V_{E} & =2 \times \int_{0}^{R-\delta} \pi x^{2} \cdot \mathrm{d} y \\
& =\frac{2 \pi}{3}(R-\delta)\left[2(U+R)^{2}+a^{2}\right] .
\end{aligned}
$$

\subsection{The condition of incompressible particles}

Since the particles can be compressible or incompressible, the data required for both estimations are different. For compressible particles, the penetration depth $\delta$ and the length from the center of the particle to the lateral surface $R^{\prime}$ are needed. For incompressible particles, however, due to particle constant 
Table 1

The initial data in the spherical and elliptical models

\begin{tabular}{llll}
\hline & & $\begin{array}{l}\text { Spherical } \\
\text { model }\end{array}$ & $\begin{array}{l}\text { Elliptical } \\
\text { model }\end{array}$ \\
\hline Incompressible particles & Ini. data & $\delta$ & $\delta, U$ \\
& Para. obtained & $R^{\prime}$ & $a$ \\
Compressible particles & Ini. data & $\delta, U$ & $\delta, U, a$ \\
& Para. obtained & - & - \\
\hline
\end{tabular}

volume, a method based on the volume balance can be utilized to carry out this calculation.

From Fig. 3, particle volume can be divided into two parts: the cylinder at the center (the contact surface as the bottom of the cylinder) and the two "shells" beside it. Combine the two terms, where the value is equal to the volume of the nondeformed particle, $\frac{4}{3} \pi R^{3}$, and the following equations can be obtained:

1. Spherical shape model:

$\frac{4}{3} \pi R^{3}=2 \pi R^{\prime 2}(R-\delta)-\frac{2}{3} \pi(R-\delta)^{3}$.

2. Elliptical shape model:

$\frac{4}{3} \pi R^{3}=2 \pi(U+R)^{2}(R-\delta)-2 \pi T^{2} \frac{(R-\delta)^{3}}{3}$,

where $T^{2}=\left[\frac{(U+R)^{2}-a^{2}}{(R-\delta)^{2}}\right]$. The initial data required for both models with compressible and incompressible cases, respectively, are listed in Table 1.

\section{Experimental}

\subsection{Material}

The particles adopted in this study are rubber and sponge particles. The rubber particle exhibits highly incompressible characteristic since its inner structure is very dense with no sufficient pores, and the sponge particle is compressible due to tiny pores distributed in it. The inner pores of the sponge particle are assumed as homogeneously distributed for simplicity. Two rubber particles with different diameters are used. The large one with a diameter of $4.8 \mathrm{~cm}$ is made of polyurethane and used for the single-particle compression test. The small one, with a diameter of $4 \mathrm{~cm}$, is made of silicon rubber and used for particle array compression with order packing. The sponge particle is $3.7 \mathrm{~cm}$ in diameter and made of polyurethane. The sponge particle inner porosity is 0.8 . Both rubber and sponge spheres were produced by molding.

\subsection{Apparatus}

\subsubsection{Measuring the particle shape with compression}

The static compression module schematic diagram, utilized to measure lateral surface profile, is shown in Fig. 4. The particles are placed on the platform. A rotator with a flat slab linked at the bottom is conducted to compress the particles, and the compressed particle images in static states can be captured via a digital camera.

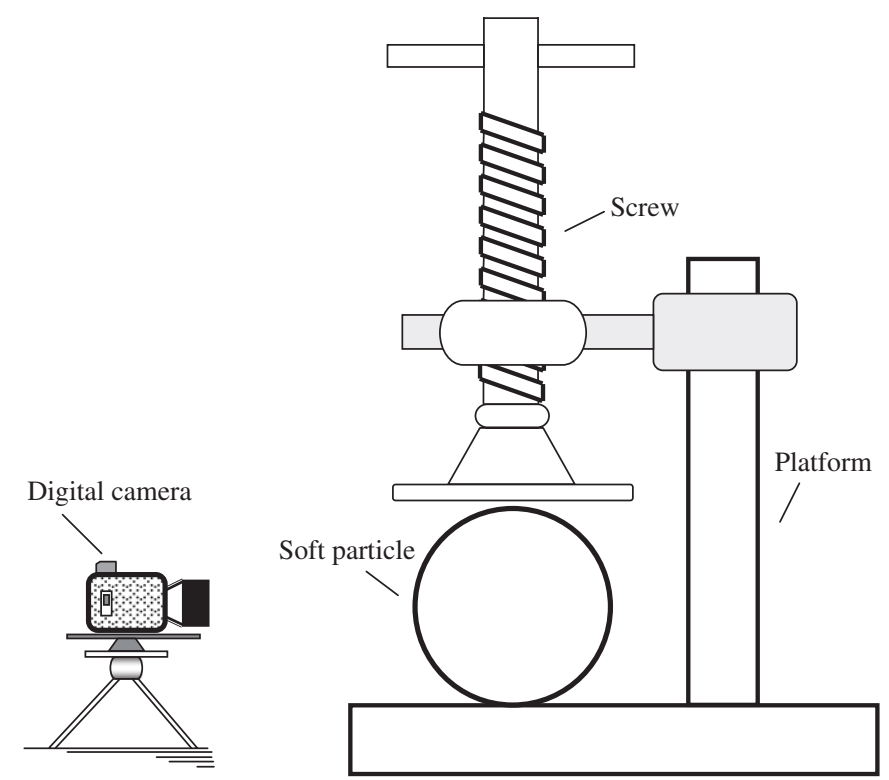

Fig. 4. The schematic diagram of the static compression unit.

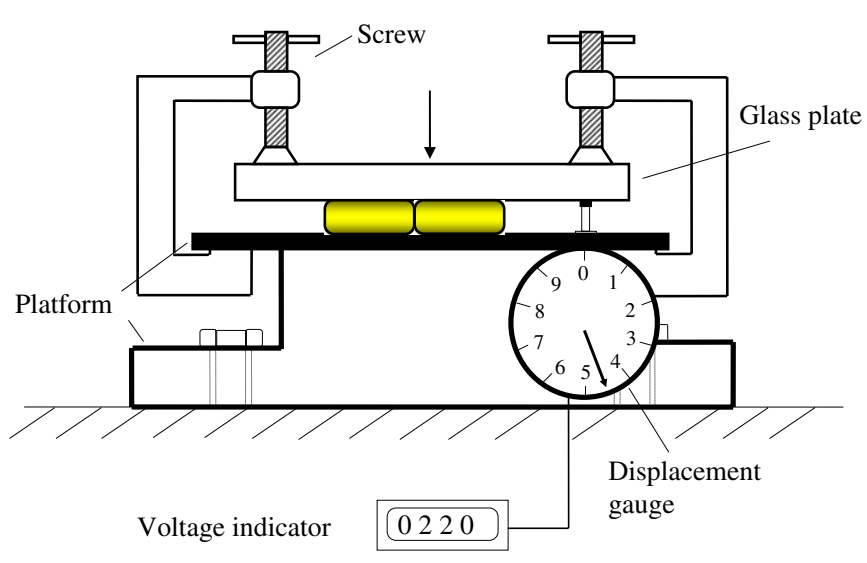

Fig. 5. The schematic diagram of planar compression unit.

\subsubsection{Two-dimensional compression module}

To examine how the lateral extensions of the compressible and incompressible particles influence surrounding particles in order-planar packing under mechanical compression, and to observe the variation in the packing structure, a two-dimensional compression instrument is used, and the schematic diagram is shown in Fig. 5. A piece of the square transparent glass slab hung by springs is fastened at the four slab corners, and the images of the compressed particle can be captured by a video camera through the glass. The particles are order packed and placed on a platform. A displacement gauge set at the platform is used to detect the penetration depth.

\subsection{Method}

\subsubsection{The compression of the particles}

Both single particle and particle-array compression are identical in process. The flat slabs used to contact the particles are 
positioned to the desired height for a specific compression ratio and then fixed. A digital camera is used to photograph the lateral shape of the particle in the single-particle compression test, and also the shape variation in the particle array at the top view through the glass slab in the particle-array compression test. The images are analyzed to obtain the information about the deforming behaviors.

\subsubsection{Variation in the inner porosity of the sponge particle}

The inner porosity measurement of sponge particle is according to the following steps: (1) Immerse the particle in water to absorb it into the pores. Repeat the process till the particle is filled up, and weigh the particle. (2) Compress the particle to a specific compression ratio, and inner water will be squeezed out. (3) Weigh the particle. The amount of water squeezed out is equal to the reduced porosity, and particle porosity variation can be determined.

\section{Result and discussion}

In this fundamental analysis, in order to model geometric property variation and investigate different compression behaviors between compressible (variable volume) and incompressible (invariable volume) soft spherical particles, the force-displacement relations, lateral extension, lateral profile of the compressed particles have been conducted experimental and discussed in this section. The compression of the order-packing particle arrays has also been analyzed to extend the finding to applications, say chromatography and filtration applications, etc.

\subsection{Force-displacement relations of the particles}

The force-displacement relations of the particles are shown in Fig. 6. For both the rubber and sponge spheres with compression ratio less than $10 \%$, the curves approximately follow Hertz law, which the force is proportional to $\frac{3}{2}$-power of compression ratio. With greater deformation, the relation diverged from Hertz theory. For the rubber sphere, it can be fitted approximately with the power of 2 and 3 for sponge sphere. Shima et al. (1993) studied the large deformation of rubber spheres and indicated that the value of power varied from 3 to 5 . In the present study, although approximately the same value can be obtained for the sponge sphere, time-delayed phenomena apparently occurred during the test as the compression ratio was greater than $10 \%$. This behavior shows that the sponge sphere is with viscoelastic character.

\subsection{Lateral extension comparison between compressible and incompressible particles}

Lateral extension comparison between compressible (sponge) and incompressible (rubber) particles under uni-axial compression is shown in Fig. 7. The pictures of the compressible particle are enlarged to the equal axial thickness with those of the incompressible one for more precise comparison. The dash lines in the pictures of the incompressible particle indicate compressible particle shapes with identical compression ratio which is defined as the ratio of the penetration depth to the diameter of non-compressed sphere. It is shown that there is greater lateral extension for the incompressible particle than that of the compressible one. This can be attributed to the more sufficient volume migration of the compressed portion to the non-contact portion in an incompressible particle than that in a compressible one, since the particle inner pores can decrease transmission efficiency of the applied loading through the particle structure in a compressible particle, and thus the migration of the particle volume can be limited. Fig. 8 shows lateral extension variation with a different compression ratio obtained from the image analysis, where the a

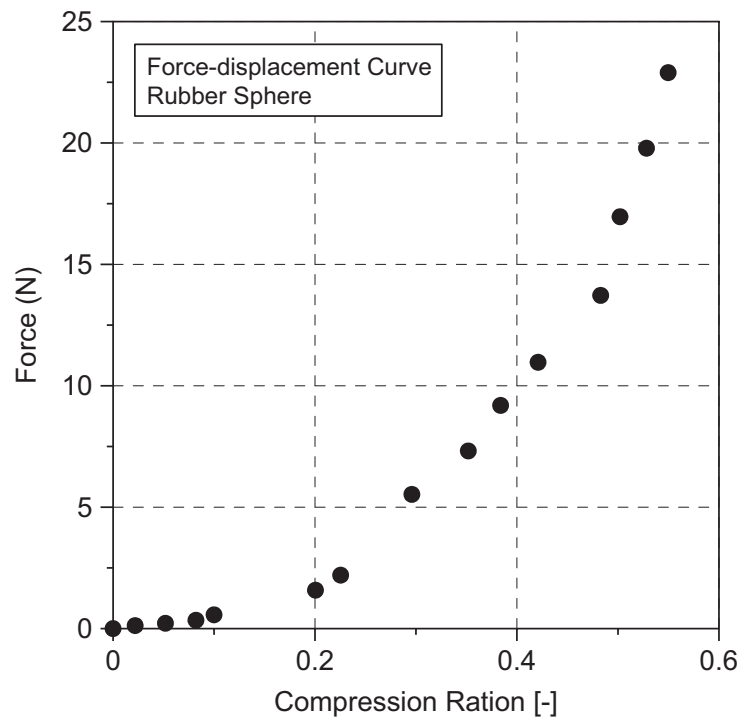

b

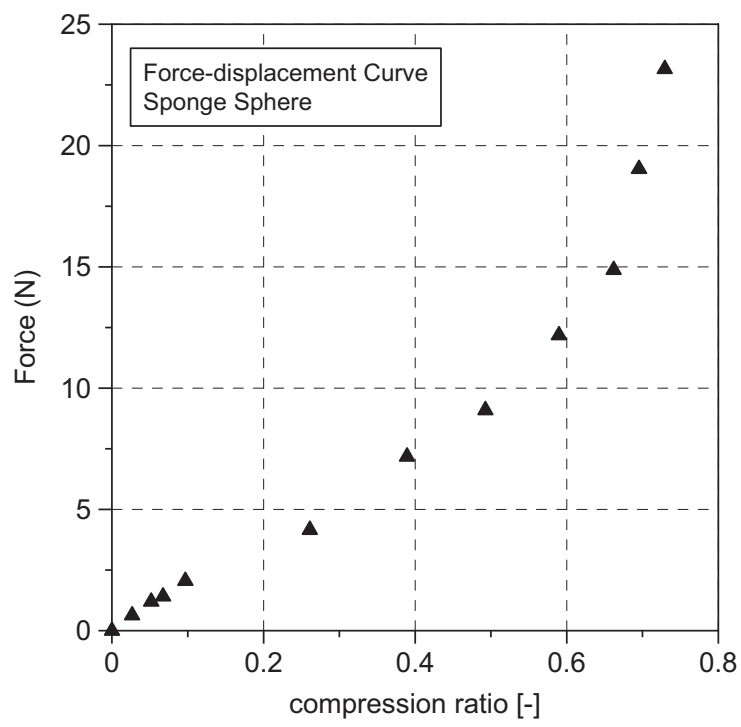

Fig. 6. The force-displacement curves for both the rubber and sponge spheres: (a) rubber sphere; (b) sponge sphere. 
a
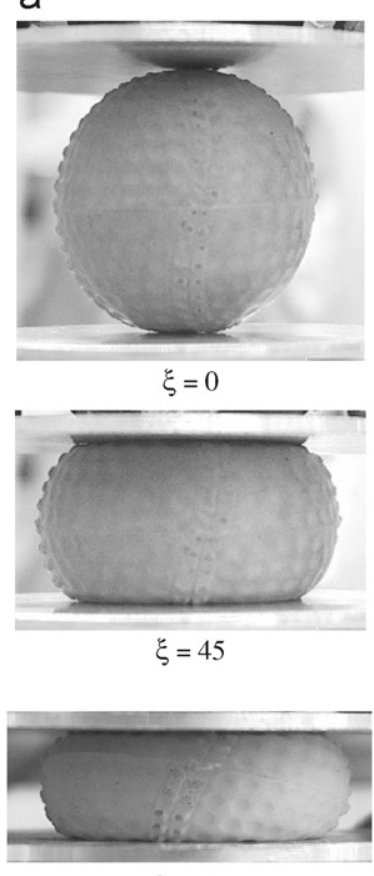

$\xi=60$ b

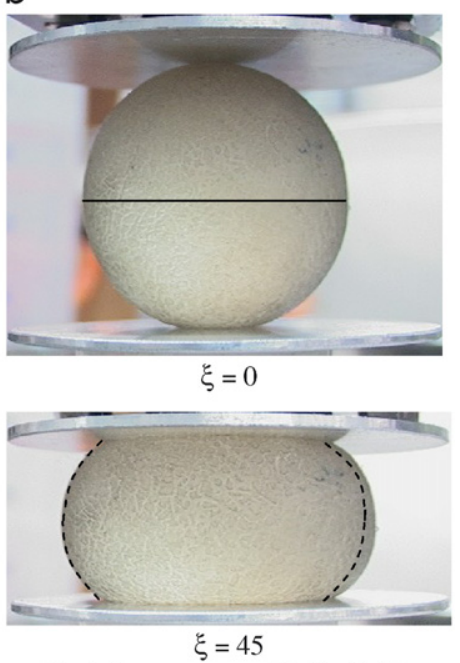

(dash line: sponge with $\xi=0.45$ )

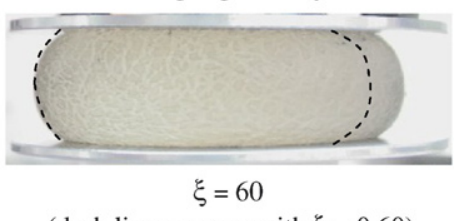

(dash line: sponge with $\xi=0.60$ )

Fig. 7. The pictures of sponge and rubber spheres in various compression stages (dash lines indicate the shape of the sponge spheres): (a) sponge sphere; (b) rubber sphere.

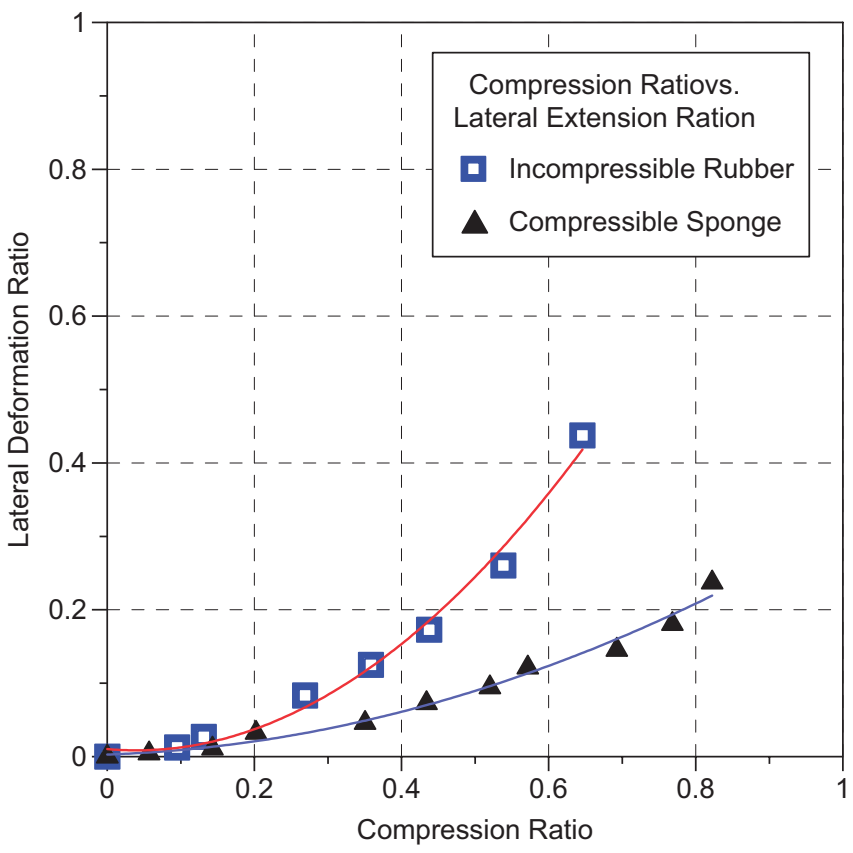

Fig. 8. The comparison between sponge and rubber spheres in the lateral/axial deformation ratio under the uni-axial compression.

lateral deformation ratio is defined as the ratio of overall lateral extension to the diameter of uncompressed particle. The degree of the deviation of the lateral extension between

a

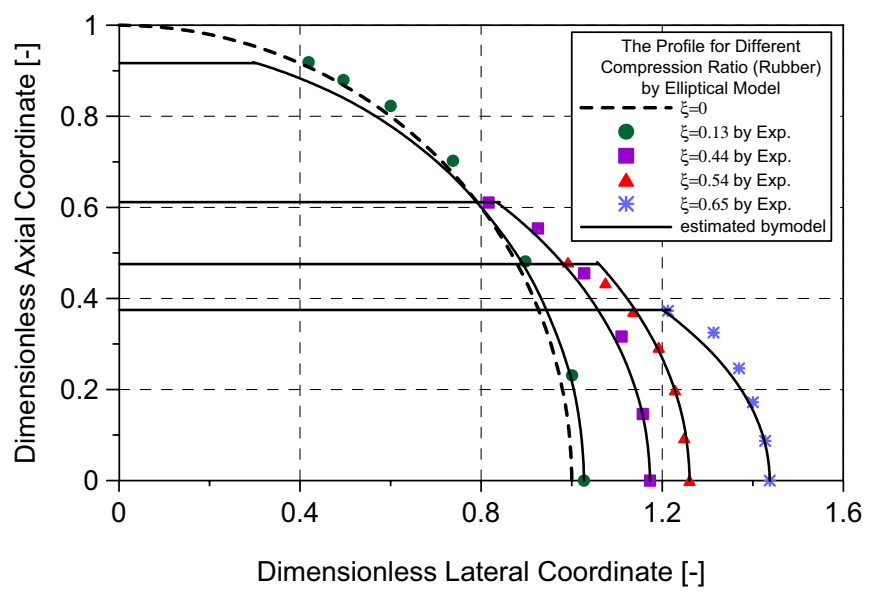

b

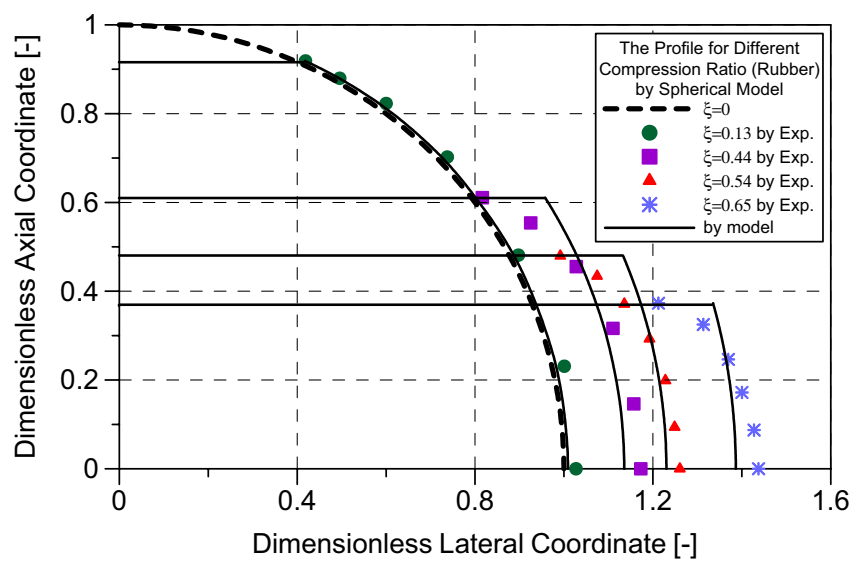

Fig. 9. The comparison between experimental and calculated lateral profiles of the compressed rubber: (a) elliptical model; (b) spherical model.

compressible and incompressible particle can be differentiated more obviously.

\subsection{The lateral profile of the compressed particle}

Figs. 9 and 10 depict the calculated lateral profiles of the compressible and incompressible particles by the proposed spherical and elliptical models, and the results are compared with the experimental values. Both the predicted curves are in good agreement with the experimental data, but the elliptical model can apparently provide better fitness. The result may be due to the following occasion: since the variation in the contact area is mainly subjected to compression unit depth loading to the particle, there is a greater extension magnitude hindrance from the lateral surface center to the portion close to the contact surface. Therefore, the extension magnitude is not uniform around the lateral surface, and the lateral surface is not able to retain spherical shape during compression, when the largest extension is in the lateral surface center. However, with a low-level compression ratio, the spherical model can provide good fitness with the experimental data. This can be inferred that the lateral surface does not vary severely with 
a

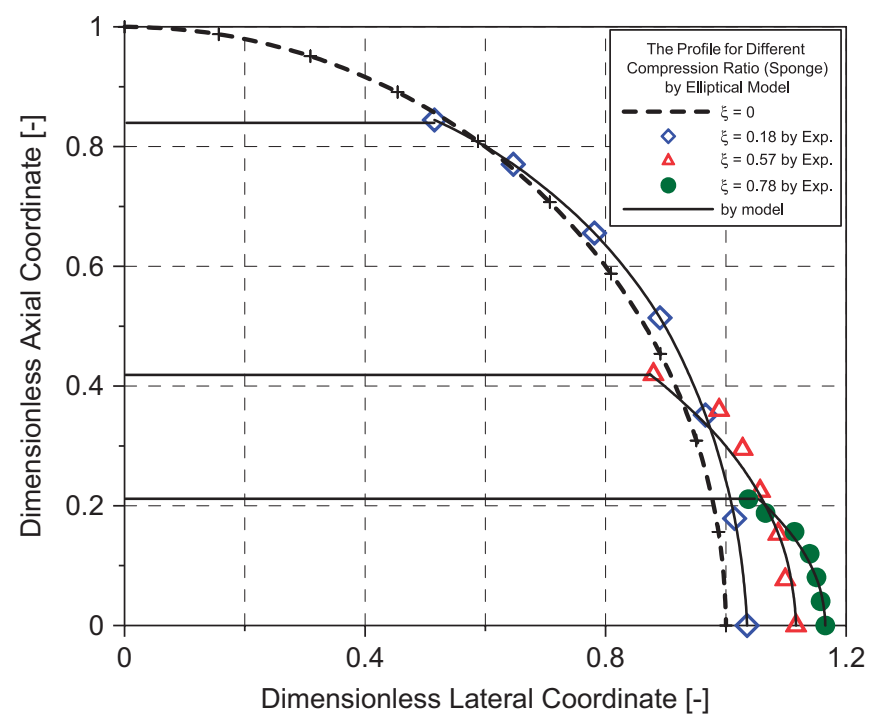

b

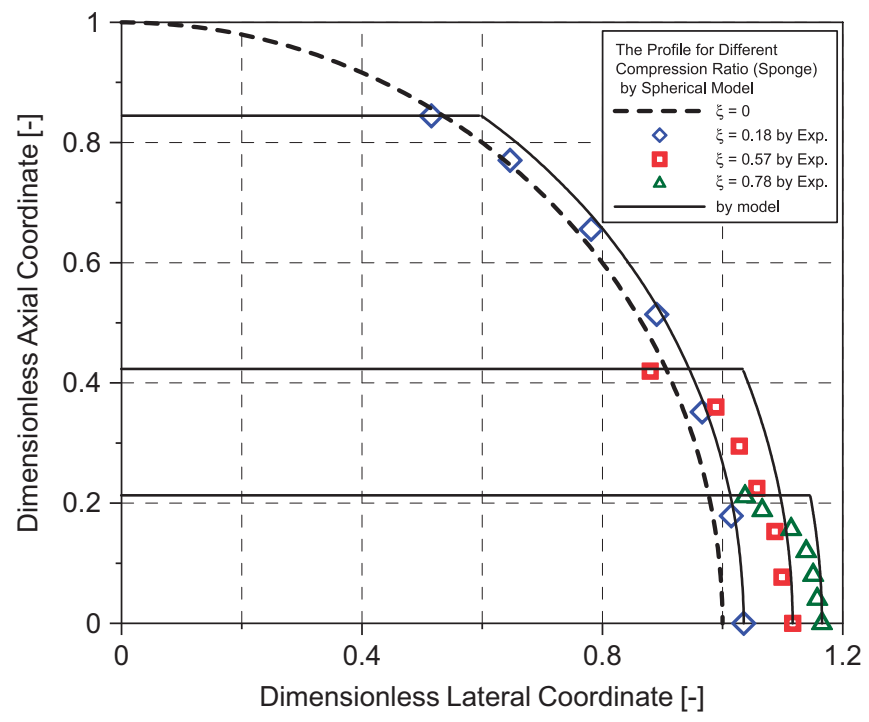

Fig. 10. The comparison between experimental and calculated lateral profile of the compressed rubber (based on lateral central width): (a) elliptical model; (b) spherical model.

low deformation degree and consequently the shape may be kept approximately spherical.

\subsection{The properties calculated by the models}

From previous discussions, the geometric models can offer fairly consistent estimation of soft particle lateral profiles with or without compression. More physical properties of the compressed particles can be determined by the equations derived above. Fig. 11 shows the calculated internal porosities of the compressible particle under various compression ratios by different geometric models and are compared with the experimental value. The calculation is based on Eqs. (5) and (13) to obtain particle volume for different models, and then subtract the volume of the solid portion measured by the experiment to

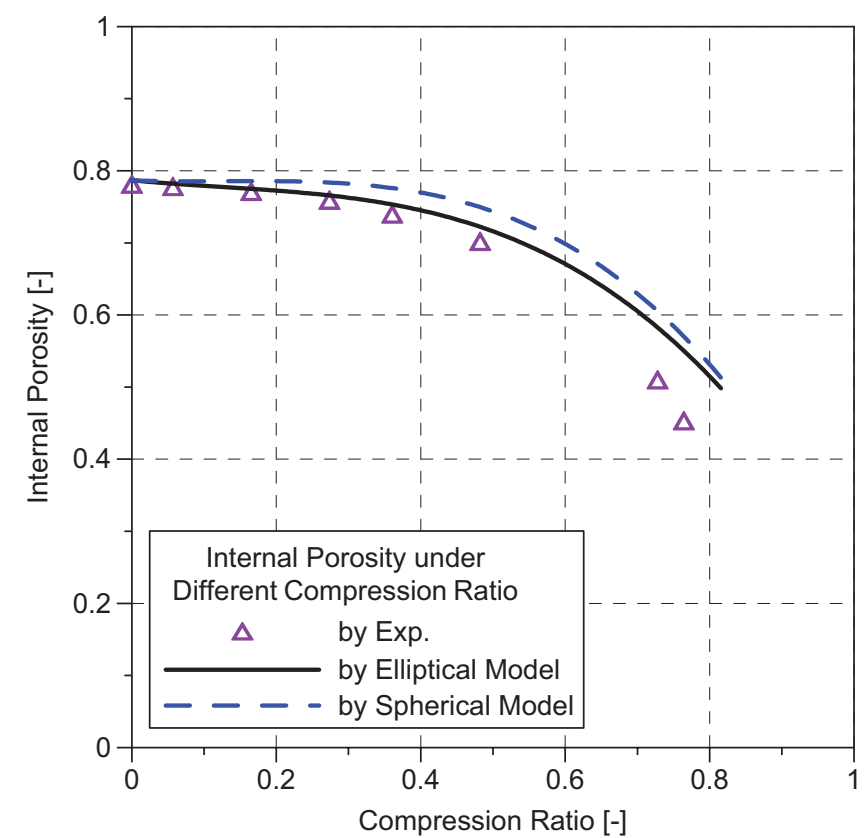

Fig. 11. The variation in internal porosity of the sponge sphere during compression.

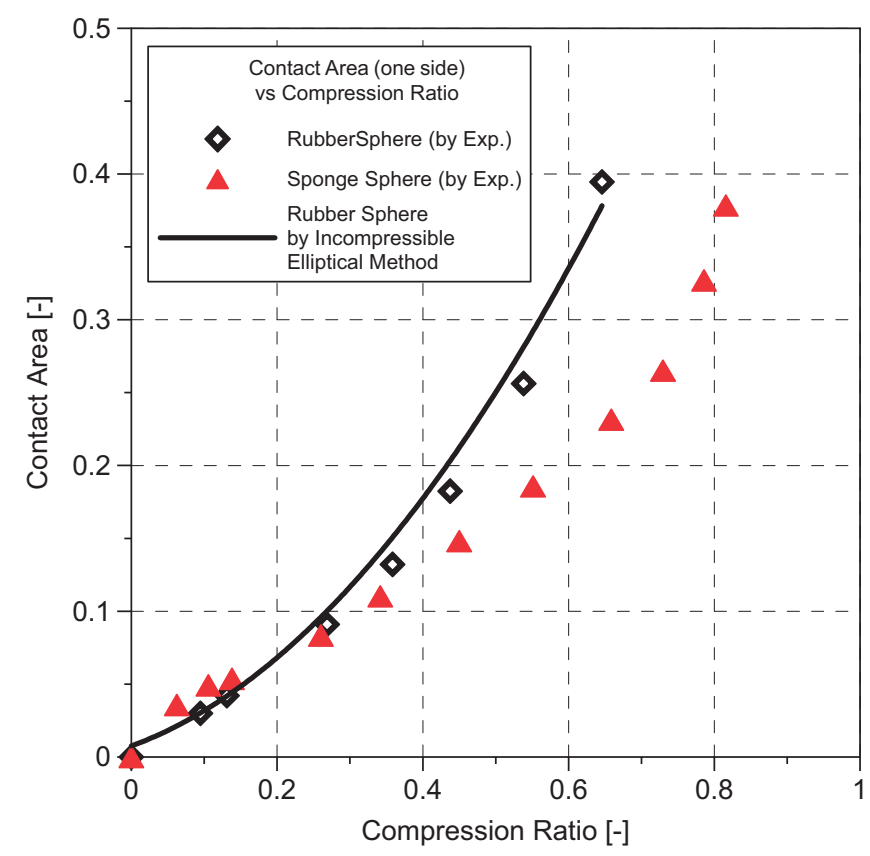

Fig. 12. The comparison of variation of contact area under various compression ratios between rubber and sponge spheres, and the value obtained by incompressible-sphere method of elliptical model.

obtain the void volume. The values calculated by the elliptical models show quite good agreement with the experimental results. The deviation between experimental and calculated data may be due to experimental limitations about the measurement of the true solid volume of the particle. Using the measurement method in the above section, all internal particle pores may not be completely filled up by water so that the amount of water 
squeezed out is not equal to the actual amount of the reduced pores during compression. As the compression ratio increases, more pores will be compressed and reduce the pore size more severely, and non-filled pore influence will increase since more pores are involved. Consequently, as the compression ratio increases, the deviation between the experimental and calculated values becomes greater as well.

Fig. 12 shows the comparison of the contact area (normalized by particle surface area in the non-compressed state) between sponge and rubber particles by experiment, and also the value calculated by the incompressible method (Eq. (15)), for the rubber particle. Since lateral surface extension of the incompressible particle is more sufficient than that of the compressible particle, the growth of the contact area under a

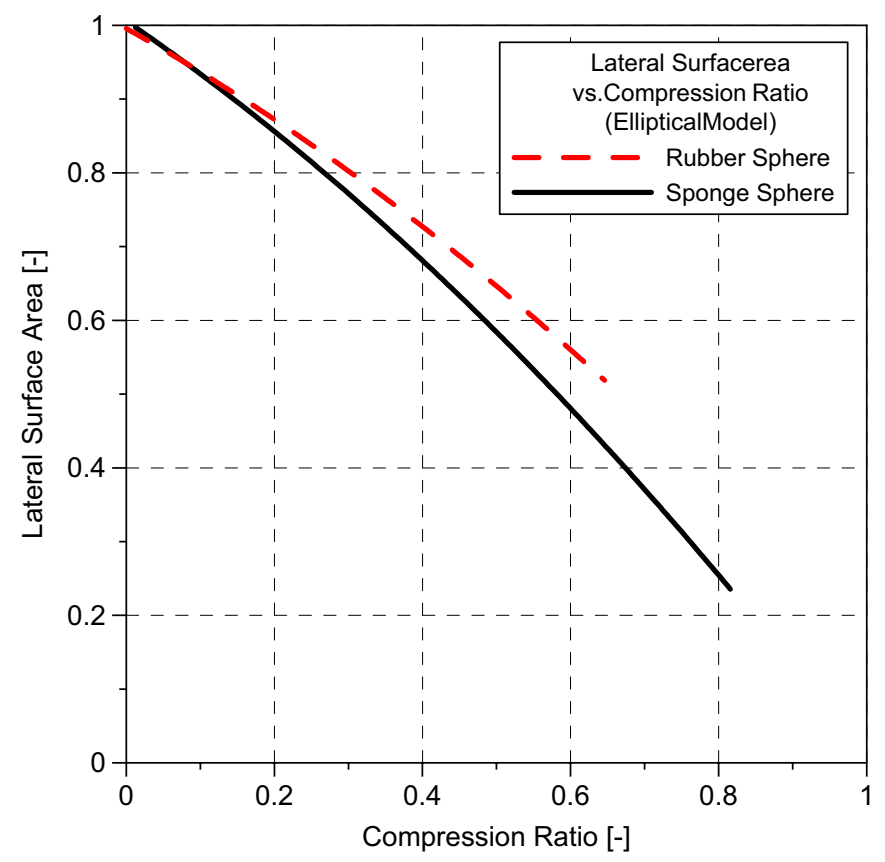

Fig. 13. The comparison of variation in lateral surface area estimated by the elliptical model under various compression ratios between rubber and sponge spheres. specific compression ratio is greater for the incompressible one. The calculated and experimental values for the rubber particle also verify that the method for the incompressible is valid. Comparison of the calculated lateral surface area between the sponge and rubber particles, which are normalized by the particle surface area in the non-compressed state, is shown in Fig. 13. The lateral surface area exhibits decrement with the compression ratio. The result also indicates that the lateral surface reduction rate is greater for the sponge particle. Since lateral surface extension is smaller for the compressible particle than the incompressible one under a specific compression ratio, lateral surface expansion will be hindered as well.

\subsection{The compression of the order-packing particle arrays}

Images of the compressed sponge and rubber particle arrays (closed packing with three particles) under equal compression ratio are shown in Fig. 14. The rubber particle image is enlarged to equal size with that of the sponge particles for a more precise comparison. Under equal compression ratio $(=0.4)$, it is very clear that the pore among the rubber particle array (porosity $\varepsilon=0.017$ ) is smaller than that of the sponge particle $\operatorname{array}(\varepsilon=0.035)$. The extension of the lateral surface for the incompressible rubber particles is greater than that of the compressible sponge particles, as mentioned in the previous section. The greater extension can therefore compress the pore between the particles more sufficiently to produce smaller porosity.

From the results of the compression of single particle and particle array, the deformation behaviors of the compressible and incompressible particles can be clearly differentiated. More intrinsic properties of the particles, mechanical parameters, and compressible particle inner porosity can be further considered to establish general theories for estimating soft particle geometric variation with more complicated packing types.

\section{Conclusions}

Comparing the geometric variation of incompressible and compressible particles under uni-axial compression was
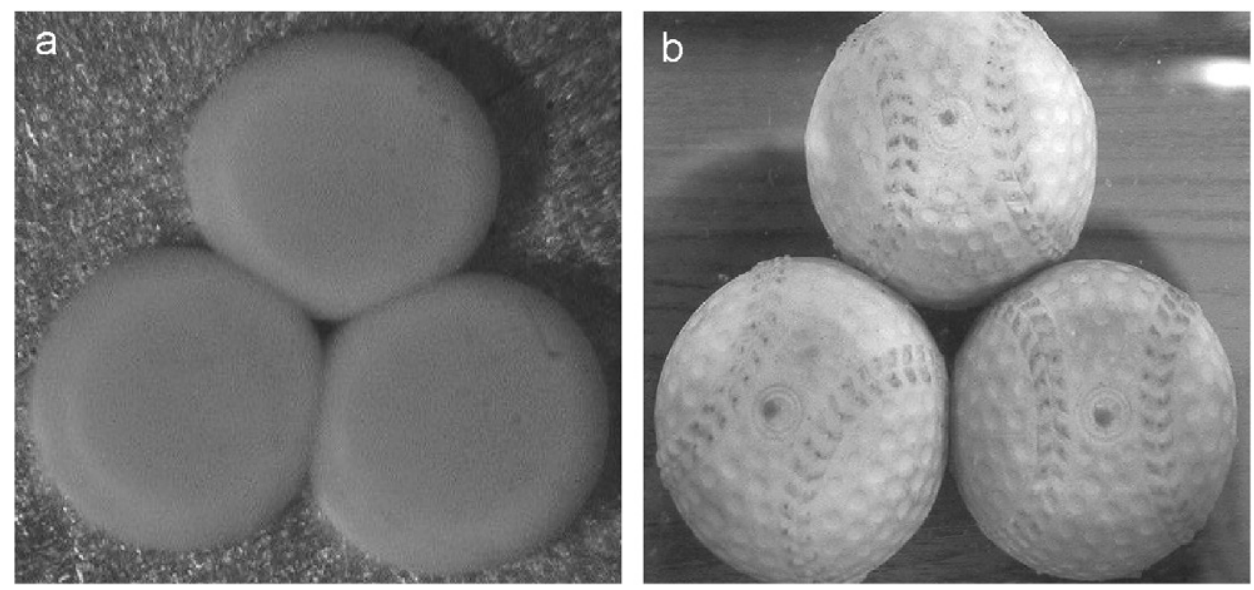

Fig. 14. The comparison of the pore size between the closest-packing spheres: (a) rubber spheres (intra porosity $\varepsilon=0.017$ ) and (b) sponge spheres (intra porosity $\varepsilon=0.035$ ). 
studied. Using the method of image analysis, compression models were proposed to estimate shape variation and particle geometric properties. Experimental results showed that there is greater lateral extension for the incompressible particle than the compressible one, since the volume of the compressed portion can migrate to the non-contact portion more sufficiently in the dense structure. Due to this characteristic, porosity between the particles shows more rapid reduction for the incompressible soft particle array with planar order packing under uni-axial compression. Lateral profiles and inner porosity variation of the compressible particle estimated by proposed models also consist well with the experimental data.

\section{Notation}

$a \quad$ radius of contact surface, $\mathrm{m}$

A lateral surface area of a compressed particle calculated by spherical model, $\mathrm{m}^{2}$

$A^{\prime} \quad$ parameter in Eq. (9a), m

$A_{E} \quad$ overall lateral surface area of a compressed particle calculated by elliptical model, $\mathrm{m}^{2}$

$A_{S} \quad$ overall lateral surface area of a compressed particle calculated by spherical model, $\mathrm{m}^{2}$

$B^{\prime} \quad$ parameter in Eq. (9b), m

$m_{0} \quad$ parameter defined in Fig. $3, \mathrm{~m}$

$R \quad$ radius of the particle, $\mathrm{m}$

$R^{\prime} \quad$ the distance from center of the compressed particle to the lateral surface, $\mathrm{m}$

$S \quad$ contact area, $\mathrm{m}^{2}$

$U \quad$ length of lateral extension of a compressed particle, $\mathrm{m}$

$V \quad$ volume of single particle after deformation calculated by spherical model, $\mathrm{m}^{3}$

$V_{E} \quad$ volume of single particle after deformation calculated by elliptical model, $\mathrm{m}^{3}$

\section{Greek letters}

$\delta \quad$ penetration depth, $\mathrm{m}$

$\varepsilon \quad$ porosity, dimensionless

$\theta \quad$ vertical included angle in Figs. 2 and 3, rad

$\xi \quad$ compression ratio, dimensionless

$\chi_{0} \quad$ the value in elliptical parameter (Eq. (9)), dimensionless

$\psi \quad$ horizontal included angle in Fig. 2, rad

\section{Acknowledgments}

The authors wish to express their sincere gratitude to the Ministry of Economic Affairs (MOEA), R.O.C. for the grant of the Technology Development Program for Academia (TDPA) project and the National Science Council (NSC) and the Centerof-Excellence (COE) Program on Membrane Technology from the Ministry of Education (MOE), R.O.C. for their financial supports.

\section{References}

Adams, M., Lawrence, C., Urso, M., Rance, J., 2004. Modelling collisions of soft agglomerates at the continuum length scale. Powder Technology $140,268-279$.

Adams, M.J., McKeown, R., 1996. Micromechanical analyses of the pressure-volume relationships for powders under confined uniaxial compression. Powder Technology 88, 155-163.

Andrei, D.C., Briscoe, B.J., Luckham, P.F., Williams, D.R., 1996. The deformation of microscopic gel particles. Journal de Chimie Physique et de Physico-Chimie Biologique 93, 960-976.

Antonyuk, S., Tomas, J., Heinrich, S., Morl, L., 2005. Breakage behaviour of spherical granulates by compression. Chemical Engineering Science 60, 4031-4044.

Devlaminck, V., 1999. A function for compressible or incompressible elastic deformation estimation. IEEE Signal Processing Letters 6 (7), 162-164.

Johnson, KL., 1985. Contact Mechanics. Cambridge University Press, New York.

Lacasse, M.D., Grest, G.S., Levine, D., 1996a. Deformation of small compressed droplet. Physical Review E 54, 5436-5446.

Lacasse, M.D., Grest, G.S., Levine, D., Mason, T.G., Weitz, D.A., 1996b. Model for the elasticity of compressed emulsions. Physical Review Letter 76, 3448-3451.

Liu, K.K., Williams, D.R., Briscoe, B.J., 1996. Compressive deformation of a single microcapsule. Physical Review E. 54, 6673-6680.

Liu, K.K., Williams, D.R., Briscoe, B.J., 1998. The large deformation of a single microelastomeric sphere. Journal of Physics D-Applied Physics 31, 294-303.

Lu, W.M., Tung, K.L., Hung, S.M., Shiau, J.S., Hwang, K.J., 2001. Compression of deformable gel particles. Powder Technology 116, $1-12$.

Reynolds, G.K., Fu, J.S., Cheong, Y.S., Hounslow, M.J., Salman, A.D., 2005. Breakage in granulation: a review. Chemical Engineering Science 60, 3969-3992.

Samimi, A., Hassanpour, A., Ghadiri, M., 2005. Single and bulk compressions of soft granules: experimental study and DEM evaluation. Chemical Engineering Science 60, 3993-4004.

Sheng, Y., Briscoe, B.J., Maung, R., Rovea, C., 2004. Compression of polymer bound alumina agglomerates at the micro deformation scale. Powder Technology 140, 228-239.

Shima, S., Tatara, Y., Ito, M., Shu, C., Lucero, J.C., 1993. Large deformations of a rubber sphere under diametral compression-part 2: experiments on many rubber materials and comparisons of theories with experiments. JSME, Series A 36, 197-205.

Tatara, Y., 1993. Large deformations of a rubber sphere under diametral compression-part 1: theoretical analysis of press approach, contact radius and lateral extension. JSME, Series A 36, 190-196.

Tung, K.L., Lin, Y.L., Shih, T.C., Lu, W.M., 2004. Fluid flow through compressible gel particles bed: a steady-state conditions. Journal of the Chinese Institute of Chemical Engineers 35 (1), 101-110. 